\title{
Thermal power treatment of step shafts
}

\author{
Alexander V. Bobrowskii ${ }^{1, *}$, Oleg I. Drachev ${ }^{1}$, Igor V. Turbin ${ }^{1}$, and Vyacheslav E. \\ Epishkin $^{1}$ \\ ${ }^{1}$ Togliatti State University, Belorusskaya St., 14, Togliatti, Russian Federation, 445667
}

\begin{abstract}
This article reviews the design of thermal power units for performing thermal operations of hardening and tempering of nonrigid parts of the "shaft" type. The authors describe the thermal power treatment technology, the residual deformation analysis and the optimal conditions definition. The units operation principles and algorithms are given. The developed technology and the design of the frame for thermal operations make it possible to maintain accuracy throughout the term of the product use.
\end{abstract}

\section{Introduction}

Many of the modern units, machines and devices contain critical non-rigid parts (NRP) of the "shaft" type; the growth of these parts is associated with the growth of the machines and mechanisms speeds. The ever-increasing number of NRP is propelled by such factors as the improvement of strength calculations, the optimization of the forms of parts and designs, the constant decrease of the products' metal intensity and the growing production of precision machines.

The disproportion in the NRP design parameters creates significant technological difficulties in manufacturing; the main reasons for these difficulties are the following:

- substantial elastic and plastic deformations throughout all the stages of the parts' processing, assembly and use;

- prominent influence of technological heredity on performance reliability;

- workpieces distortion caused by uneven residual stresses introduced at all stages of processing.

The negative effect of these factors during the NRP manufacturing leads to the disruption of technological bases, to the errors in the parts' shape and size and, eventually, to the overall decrease of performance reliability of NRP and finished products.

\section{Importance and goal of this research}

The modern technology that ensures the obtainment of high-quality products makes provisions for solving complex and interrelated problems. A number of key issues connected to ensuring the high quality of parts manufacturing is being successfully solved thanks to the works by numerous research teams and scientists. However, there are

\footnotetext{
* Corresponding author: ba838@yandex.ru
} 
problems which, in spite of being quite significant, are still not studied enough. One of them is the problem of struggle against the distortion of parts during their processing, caused by the effect of residual stresses [1-3]. Almost all the technological processes of manufacturing various mechanical parts lead to the appearance of residual stresses.

The goal of development of this technology is to increase the operational accuracy of long non-rigid step shafts. In order to achieve that, the step frame is installed onto the shaft workpiece by sections: the first one is central step of the workpiece, which is fixed on both sides relative to the frame section using spherical pivots; and then the following frame sections are installed on both sides of the central section one after another. It creates the uniform internal stress of the workpieceby selectingequal ratiosof the cross-section areas of the frame sections and the corresponding workpieces steps. The preselected value of the plastic deformations of the workpiece step is created by selecting the length of the frame section and the height of the cup-shaped covers-baseplates.

\section{Basicreasoning}

The essence of the technological solutionis to change the processing sequence, unlike the known methods [4], where equal plastic deformationand axial stresseswere appliedover the entire length of the workpiece.The workpiece itself is smooth; in the proposed variant the plastic deformation, as well as working stressesare applied afterrough mechanical processing, when the longitudinal and the transverse profiles of each step of the workpiece are formed.At that, the stresses are kept consistent and equal along the length and in any section of the workpiece. When the workpiece is cooled, taking into consideration the unloading theorem, the residual stresses equal at all sections of the shaft workpiece are formed throughout the workpiece volume; which makes it possible to eliminate the distortion of long workpieces.

The described processing method of thermal power treatment is based on the studies of thermal treatment technology and metal technological heredity [5-8], as well as on the theory of thermo-elastic-plastic phenomena occurring in a metal.

The process of applying this technology consists of the following technological operations. The shaft workpiece is turned on a lathe machine with the use of self-centering steady rests, its operating profile is formed. Threads are cut at the ends of each step,and the thread depth(its inside diameter)should not exceed the allowance for the finish machining (turning or polishing).

After that radiators 2 (Fig.1) with radial grooves are put onto the central step of the frame, where workpiece 1 has the maximum cross section, and are fixed along the length with a calculated pitch.

The number of radiators 2 and their effective area are estimated by the required heat removal surface. The size of the radiators' area is regulated by the number of grooves proportionally with the workpiece working surface. A set of the radiators with various heat removal surfaces ensures the uniform cooling of the workpiece along the cross section and length of the workpiece. The latter can have a complex form in longitudinal and cross sections (Fig. 1). The workpiece with radiators 2 is installed into the multi-layer assembled frame 3 made with through holesalong the length and the diameter of the frame. Each layer of the frame is filled with filler; the latter, depending on the required cooling rate of the frame, may have various thermal conductivity coefficients (cast iron chips, gravel sand and so on). After that the central step of the workpiece (with the maximum cross section) is fixed relative to the end planes of the central section of frame 3 with the help of coversbaseplates 4 and spherical pivots 5; the pivots are screwed to the ends of the central step until tight with covers-baseplates 4 , thus creating the closed power circuit workpiecespherical pivots - covers-baseplates - frame. 

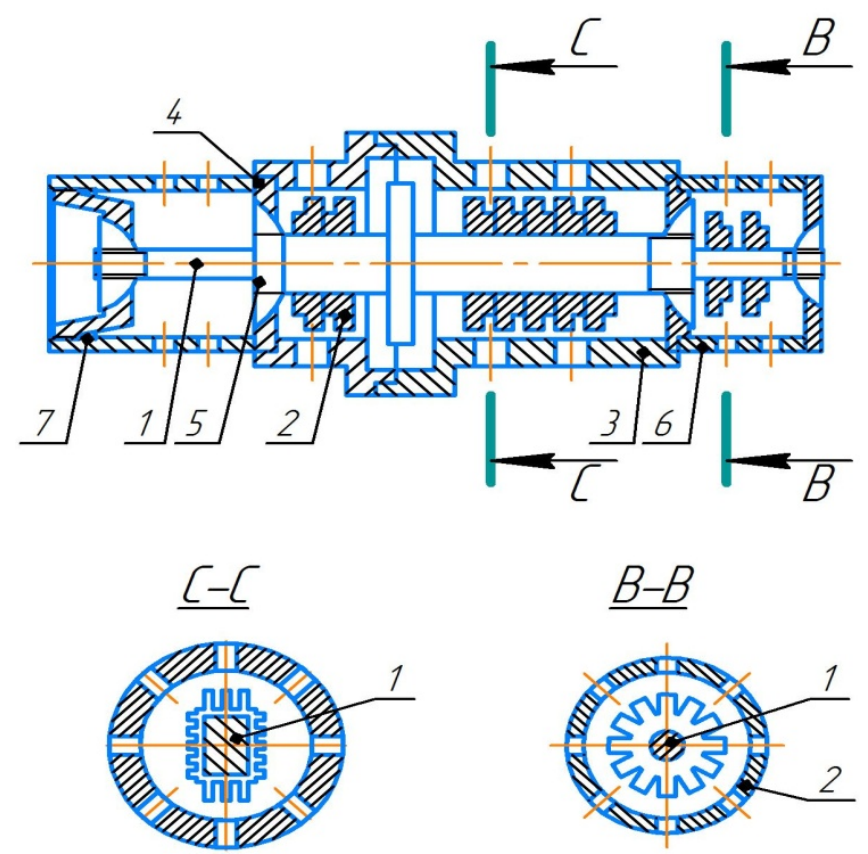

Fig. 1. Assembled frame with the workpiece installed into it.

If the workpiece has a hub with a diameter much larger than the maximum diameter of the workpiece, then the frame section where the hub is located is made to be assembled. Then sequentiallyradiators 2are put on and fixed on the left and right parts of the workpiece, on its steps (the number of the radiators and their heat removal surface are calculated, like for the central step of the workpiece, in such a manner that the workpiece cooling is uniform both along its length and cross section). After that, sections 6 and covers-baseplates 4 are put and spherical pivots 5 are screwed; after that the assembly is the same for all the steps of the workpiece. The areas of the cross sections of the frame are calculated according to the specified cross-sectional areas of the workpiece steps. The ratio of the cross-sectional areas of the frame sectionsto the corresponding cross-sectional areas of the workpiece should be constant and equal to each other at all steps of the shaft. The latter ensures the constancy of the working stress when the workpiece is extended and compressed, heated and cooled, which leads to the formation of equal and constant residual stressesover the cross section and along the length of the workpiece.With a decrease of rigidity of the workpiece steps, the values of distortion of these steps are always greater than the rigid parts of the workpiece. That is why it is necessary to increase the values of deformations for these parts of the workpiece in order to select the amounts of deflections and to perform the axial plastic deformations. In order to do that, the threads are cut on the outer diameters of the covers-baseplates 4 or 7 (the latter are made to be cup-shaped to be regulated by the value of plastic deformation) and the corresponding internal threads are cut in frame sections 10. Cover-baseplate 7 is screwed into the frame section according to a calculated value, thus ensuring the elimination of the amount of deflection and the value of the plastic deformation of this step of the workpiece. The assembled structure is lowered into a shaft furnace and heatedaccording to the thermal treatment technology up to the temperature of hardening or tempering and is kept at this temperature until the workpiece is completely heated.When heated, the frame lengthens more than the workpiece; its linear 
expansion coefficient is greater than the workpiece linear expansion coefficient. The value of the workpiece plastic deformation exceeding the proportional limit is obtained through the selection of the numerical values of the positive difference between the linear expansion coefficients of the frame and the workpiece.

The axial plastic deformation beyond the proportional limit (Hooke's law) during the heating eliminates technological heredity from previous operations and at the same time corrects workpieces. However, the non-uniform mode of cooling in a free state causes its own non-uniformresidual stresses; in order to avoid this negative technological phenomenon, it is necessary for frame 3 to cool slower than workpiece 1 (i.e. the workpiece must cool at least five times faster than the frame), depending on the cooling medium: oil, water, air. During the cooling with axial loading, the residual stresses, which are uniform along the length and at cross section, are formed in the step workpiece; that eliminates the distortion of finished shaft parts during their use. Beside that, the strength characteristics of the workpiece are increased. The frame is made of steel 12X18E10T, the shaft workpiece is made of steel $30 \mathrm{X} 13$, the temperature extensions are calculated by the formula:

$$
\Delta l=\alpha_{\text {frame }}\left(T^{\circ}\right) \cdot T^{\circ} \cdot L-\alpha_{\text {part }}\left(T^{\circ}\right) \cdot L
$$

where $\Delta l$ is the difference between the extensions of the frame and the shaft; $\alpha$ is the linear expansion coefficient; $\mathrm{T}^{\circ}$ is the heating temperature and $\mathrm{L}$ is the length of the shaft and the frame.

When $\mathrm{L}=1000 \mathrm{~mm}, \mathrm{~T}^{\circ}$ is $850{ }^{\circ} \mathrm{C} ; \alpha_{\text {frame }}=1 /$ degree, $a$ part $=1 /$ degree, the plastic deformation value is $e=0,84 \%$.The calculation of the cooling time is performed according to the following parameters: shaft diameter is $60 \mathrm{~mm}$, the frame inside diameter is $210 \mathrm{~mm}$, the outer diameter is $310 \mathrm{~mm}$, the filler is sand mixed with cast iron chips, the cooling medium is oil at the temperature $30^{\circ} \mathrm{C}$. The shaft cooling time is calculated by the formula:

$$
r=\left(F^{\circ} d^{2} c \gamma\right) c / 4 \lambda
$$

The time of frame cooling is defined by the specific solution of the equation

$$
\mathrm{Q} / \mathrm{Q}^{\prime}=\mathrm{F}_{0}\left(\mathrm{~B}_{\mathrm{i}} \mathrm{F}_{0}\right)
$$

where $\mathrm{F}_{0}=\mathrm{a}^{\mathrm{r}} / \delta^{2}$ is the Fourier criterion, which is defined as the function of relative temperatures $Q / Q^{\prime}$ and the criterion $B_{i}$, the latter is equal to $B_{i}=C / \lambda_{\text {eq }} ; \lambda_{\text {eq }}$ is the equivalent thermal conductivity of the two-layer wall of the frame; $\gamma$ is the material volume weight; $\mathrm{C}$ is the coefficient of heat emission from the environment to the surface of the body; $a$ is the thermal diffusivity coefficient, $\delta$ is the radius of a long product.

The step workpieces are calculated on the basis of equality of the generated working stresses at all steps of the workpiece. The stresses in any steps of the workpiece should be equal, i.e.:

$$
\sigma_{n}=\sigma_{n-1}
$$

Here $\sigma_{n}$ and $\sigma_{n-1}$ are the working stresses in $\mathrm{n}$ and (n-1) steps of the workpiece.

The tensions developed by the frame sections are defined by the formula:

$$
P_{n}^{f r}=F_{n}^{f r} \cdot \alpha\left(T^{\circ}\right) \cdot T^{\circ}
$$


where $F_{n}^{f r}$ is the area of the frame cross section, $E_{n}^{f r}$ is the frame material elastic modulus; $\alpha\left(\mathrm{T}^{\circ}\right)$ is the difference between the linear expansion coefficients of the workpiece and frame material; $\mathrm{T}^{\circ}$ is the frame temperature.

The axial expansion working stresses in $\mathrm{n}$ and (n-1) steps of the workpiece are defined by the equity:

$$
F=\frac{F_{n}^{f r} \cdot \alpha\left(T^{\circ}\right) T^{\circ}}{F_{n}}=\frac{F_{n-1}^{f r} \cdot E_{n-1}^{f r} \cdot \alpha\left(T^{\circ}\right) T^{\circ}}{F_{n-1}}
$$

Here $F_{n}$ and $F_{n-1}$ are the cross section areas of the workpiece in $\mathrm{n}$ and (n-1) steps.

\section{Experimental results.Conclusion}

The experimental studies carried out with the help of the samples made it possible totest the proposed method inworkshop conditions. The shaft workpieces made of steel 30X13 were treated, and the frame was made of steel12X18H10T. The geometric parameters of the workpiece were the following: its length was $3400 \mathrm{~mm}$, its diameter was $45 \mathrm{~mm}$, the length of the finished part was $3200 \mathrm{~mm}$, itsdiameter was $38 \mathrm{~mm}$. The temperature mode of thermal treatment was not changed: the hardening temperature was $\mathrm{T}=1000 \ldots 1050^{\circ} \mathrm{C}$, followed by cooling in air.The runout of the workpiece and the finished parts was measuredin ten sections along the entire length; at that, the product was placed on two prisms in such a manner that the shaft was divided into three equal parts.After cooling in air, the runout was checked in ten sections, at the same time the hardness was measured along the length of the product.

The thermal power treatment (hardening) with the help of the developed unit provided positive results, the shaft runout was not more than $10 \mu \mathrm{m} / \mathrm{m}$ and did not exceed the allowance for turning. The hardness dispersion did not exceed $12 \%$ at the maximum. It should be noted that according to the traditional technology of shaft treatment, the hardening was performed in a freely suspended state; in that case the shaft runout was within 7-8 mm.

It should be specifically noted that after hardening with the use of the thermal power treatment the plane of maximum deformations was within $40-45^{\circ} \mathrm{C}$, which indicates the presence of high tangential stresses in the workpiece. Unfortunately, this was not taken into account in thetheoreticalinsights by the authors [12].

The application of the proposed technological method allows to minimize the runoutof the shaft and to stabilize the level of residual stresses along its length. The latter allows to increase the exploitation accuracy and the quality of the finished products.

\section{References}

1. V. Lokhov, Y. Nyashin, F. Ziegler, Statement and solution of optimal problems for independent stress and deformation control by eigenstrain. Zeitschrift fur Angewandte Mathematik und Mechanik, Vol. 89, No 4, pp. 320-332, 2009.

2. A. Gruening, M. Lebsanft, B. Scholtes, Residual Stress State in Tools Used for Thermo-mechanical Metal Forming Processes.Engineering Applications of Residual Stress, Volume 8. Part of the series Conference Proceedings of the Society for Experimental Mechanics Series, pp. 39-45, 25 May 2011. 
3. J. Dong, J. Epp, A. da Silva Rocha, R. Menezes Nunes, H. Werner Zoch, Investigation of the Influence Factors on Distortion in Induction-Hardened Steel Shafts Manufactured from Gold-Drawn Rod. Metallurgical and Materials Transactions A, pp. 1-12, 25 November 2015

4. O. Drachev, D. Rastorguyev, Laboratory tests to evaluate the influence of the thermal power treatment parameters on the shafts distortion. Automation of technological processes and production control, pp. 123-129 (Togliatti, TGU, 2000).

5. G. Hoziruk, Influence of the residual stresses in a workpiece on the form error of nonrigid parts of the plate type. Improvement of technological processes in machine manufacturing, pp. 137-140 (Irkutsk, 1987).

6. L. Kolot, Development and research of the set of technological methods of improvement of the form accuracy of non-rigid parts, p. 19 (Minsk, 1986).

7. P. Yascheritsin, Y. Skorypin, Technological and exploitation heredity and its influence on machines endurance, p. 246 (Minsk, Nauka I Tekhnika, 1978).

8. A.A. Pozdeev, Residual stresses: Theory and applications / A.A.Pozdeev, Y.I. Nyashin, P.V. Trusov (Moscow, Nauka, p. 112 (1982) 